\title{
The oncogene Etv5 promotes MET in somatic reprogramming and orchestrates epiblast/primitive endoderm specification during $\mathrm{mESCs}$ differentiation
}

Jinglong Zhang ${ }^{1}$, Hongxia Cao ${ }^{1}$, Jing Xie ${ }^{1}$, Chen Fan ${ }^{1}$, Youlong Xie ${ }^{1}$, Xin $\mathrm{He}^{1}$, Mingzhi Liao ${ }^{2}$, Shiqiang Zhang ${ }^{1}$ and Huayan Wang ${ }^{1}$

\begin{abstract}
Unipotent spermatogonial stem cells (SSCs) can be efficiently reprogrammed into pluripotent stem cells only by manipulating the culture condition, without introducing exogenous reprogramming factors. This phenotype raises the hypothesis that the endogenous transcription factors (TFs) in SSCS may facilitate reprogramming to acquire pluripotency. In this study, we screened a pool of SSCs TFs (BCI6b, Lhx1, Foxo 1, Plzf, Id4, Taf4b, and Etv5), and found that oncogene Etv5 could dramatically increase the efficiency of induced pluripotent stem cells (iPSCs) generation when combined with Yamanaka factors. We also demonstrated that Etv5 could promote mesenchymal-epithelial transition (MET) at the early stage of reprogramming by regulating Tet2-miR200s-Zeb1 axis. In addition, Etv5 knockdown in mouse embryonic stem cells (mESCs) could decrease the genomic $5 \mathrm{hmC}$ level by downregulating Tet2. Furthermore, the embryoid body assay revealed that Etv5 could positively regulate primitive endoderm specification through regulating Gata6 and negatively regulate epiblast specification by inhibiting Fgf5 expression. In summary, our findings provide insights into understanding the regulation mechanisms of Etv 5 under the context of somatic reprogramming, mESCs maintenance, and differentiation.
\end{abstract}

\section{Introduction}

Somatic cells can be reprogrammed into pluripotent state by overexpressing defined transcription factors (TFs) in vitro ${ }^{1}$. By contrast, spermatogonial stem cells (SSCs) endogenously express pluripotent $\mathrm{TFs}$, including Oct3/4, Sox2, Klf4, $c-M y c$, and $\operatorname{Lin} 28^{2}$. Therefore, SSCs can be efficiently reprogrammed into pluripotent state only by changing the culture condition without overexpressing exogenous $\mathrm{TFs}^{3}$. These evidences indicate the TFs involved in SSCs self-renewal may facilitate the

\footnotetext{
Correspondence: Shiqiang Zhang (shiqiangzhang@nwsuaf.edu.cn) or Huayan Wang (hhwang101@163.com) (hhwang101@nwsuaf.edu.cn)

${ }^{1}$ College of Veterinary Medicine, Shaanxi Center of Stem Cells Engineering \& Technology, Northwest A\&F University, Yangling, Shaanxi 712100, China

${ }^{2}$ College of Life Sciences, Northwest A\&F University, Yangling, Shaanxi 712100, China
}

Edited by $Y$. Shi reprogramming to acquire pluripotency. However, no study has been made systematically to investigate the roles of SSCs self-renewal TFs in somatic reprogramming.

Ets (E-twenty-six) variant gene 5 (Etv5) is one of the essential TFs for SSCs self-renewal ${ }^{4,5}$. Etv5 knockout can cause Sertoli cell-only syndrome on mouse ${ }^{4}$. Etv 5 is also suggested to regulate proliferation and differentiation of mouse embryonic stem cells (mESCs) ${ }^{6}$. In addition, Etv5 has been found as an early regulator during somatic reprogramming ${ }^{7}$. However, the exact mechanism of Etv5 in $\mathrm{mESCs}$ and somatic reprogramming remains unclear.

Ten-eleven translocation family proteins (TET1/TET2/ TET3) can oxidize 5-methylcytosine $(5 \mathrm{mC})$ into 5hydroxymethylcytosine $(5 \mathrm{hmC})$ and regulate gene transcription $^{8}$. Overexpression of Tet 1 and Tet2 has proven to promote somatic reprogramming by 
reactivating pluripotency genes ${ }^{9,10}$. Ironically, Tet1 and Tet2 are dispensable for reprogramming SSCs into pluripotency ${ }^{11}$. However, Tet 1 and Tet 2 are suggested to erase H19 imprinting locus during reprogramming ${ }^{11}$. Although extensive studies have been taken to investigate the roles of Tets proteins, the upstream regulators of Tets proteins are not completely understood.

In this study, we screened a pool of SSCs TFs and found Etv5 could remarkably promote the efficiency of reprogramming when combined with Yamanaka factors. We also proved that Etv5 could facilitate mesenchymal-epithelial transition (MET) through Tet2miR200s-Zeb1 regulation axis. Moreover, we found Etv5 knockdown (KD) could decrease the expression level of Tet 2 and $5 \mathrm{hmC}$ in $\mathrm{mESCs}$ and delayed the primitive endoderm differentiation by downregulating Gata6. In addition, Etv5-KD in mESCs could increase the epiblast specification in vitro by upregulating $F g f 5$ expression. Our findings provide insights to understand the mechanisms of Etv5 in somatic reprogramming, mESCs maintenance and differentiation.

\section{Results}

\section{Etv5 enhances somatic cell reprogramming}

We initially hypothesized that SSCs self-renewal TFs could promote the efficiency of generating induced pluripotent stem cells (iPSCs) (Fig. 1a). We screened SSCs-specific TFs by reference retrieval and constructed a pool consisting of Bcl6b, Lhx1, Foxo1 Plzf, Id4, Taf4b, and Etv5. Then we investigated their roles in promoting iPSCs efficiency. Combined with Yamanaka factors (Oct4, Sox2, Klf4, and $c-M y c$, abbreviated as OSKM hereafter), four SSCs TFs (Bcl6b, Lhx1, Foxo1 and Plzf) decreased the efficiency of iPSCs (Oct4-GFP ${ }^{+}$colonies/ $50000 \mathrm{MEFs}$ ). In contrast, three SSCs TFs (Id4, Taf4b and Etv5) increased the efficiency of iPSCs (Fig. 1b). Of note, Etv5 showed the highest efficiency when compared to Id4 and Taf $4 b$.

Then we tested whether the iPSCs generated from combination of OSKM and Etv5 (abbreviated as OSKMEiPSCs hereafter) have typically pluripotent features. OSKME-iPSCs were positive for NANOG and SSEA-1 staining (Supplementary Fig. S1a) and expressed similar levels of endogenous pluripotent genes (Oct4, Sox2, Nanog, Rex1, and Klf2) when compared to OSKM-iPSCs and J1 mESCs (Supplementary Fig. S1b, c). All the characterized cell lines of OSKME-iPSCs were integrated exogenous Oct4, Sox2, Klf4, c-Myc, and Etv5 (Supplementary Fig. S1d), but the transgenes were totally silenced (Supplementary Fig. S1e). The promoters of Oct4 and Nanog also showed dramatic DNA demethylation in OSKME-iPSCs when compared with MEFs (Supplementary Fig. S1f). The OSKME-iPSCs could also differentiate into three germ layers as assayed by embryoid body (EB) differentiation (Supplementary Fig. S1g) and teratoma formation (Supplementary Fig. S1h).

We next examined whether Etv5 could promote reprogramming when combined with OSK, but observed no significant improvement on iPSCs efficiency (Fig. 1c). Furthermore, we asked whether Etv 5 could replace anyone of other Yamanaka factors to achieve full reprogramming. However, Etv5 was unable to replace either of them (Fig. 1c).

We also investigated whether endogenous Etv5 was reactivated during OSKM-mediated reprogramming. Interestingly, a fluctuation of Etv5 expression was observed in the process of reprogramming. Endogenous Etv 5 was activated on day 4 after introducing OSKM and reached the top level during day 6-10. Then the expression level of Etv5 declined significantly (Fig. 1d). This phenotype intrigued us to determine the best time window of introducing exogenous Etv5 to get the highest reprogramming efficiency. Therefore, we compared the reprogramming efficiency which was generated by two ways: introducing exogenous Etv 5 from beginning after OSKM transduction, and 6 days after OSKM transduction. Interestingly, we found overexpression of Etv5 on day 6 after OSKM transduction could not increase the efficiency of reprogramming. Only overexpression of Etv5 from beginning together with OSKM could significantly increase the iPSCs efficiency (Fig. 1e). To further determine whether endogenous Etv 5 is essentially required for reprogramming, we performed Etv5 shRNA interference at the early stage of OSKM reprogramming, and found that $O c t 4-\mathrm{GFP}^{+}$colonies decreased significantly as the endogenous Etv5 was knocked down (Fig. 1f). Together these results indicate that Etv 5 can increase iPSCs efficiency at the early stage of reprogramming when combined with Yamanaka factors.

\section{Etv5 promotes cell reprogramming by facilitating MET}

To dissect the mechanisms by which Etv 5 promotes reprogramming, we examined whether Etv5 follows the proposed models at the early stage of reprogramming ${ }^{12}$. We considered two possibilities: (1) Whether Etv5 could increase cell proliferation? (2) Whether Etv5 could promote MET?

First, cell growth curves were drawn and compared between OSKME and OSKM. Unexpectedly, Etv5 significantly decreased the cell proliferation (Supplementary Fig. S2a). This phenotype was consistent with the downregulation of cell cycle positive regulators (Ccnd1, Ccne2, and $C d k 4$ ) (Supplementary Fig. S2b) and the upregulation of cell cycle negative regulators (Cdkn1a, Cdkn2a) (Supplementary Fig. S2c) in OSKME when compared to OSKM.

Second, we assessed if Etv5 could promote MET during the early stage of reprogramming. Immunofluorescence 
a

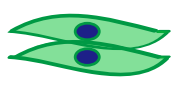

Somatic cells

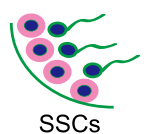

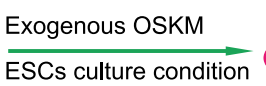

ESCs culture condition

Endogenous TFs

$\overrightarrow{\text { ESCs culture condition }}$

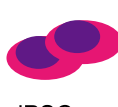

iPSCs

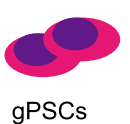

b

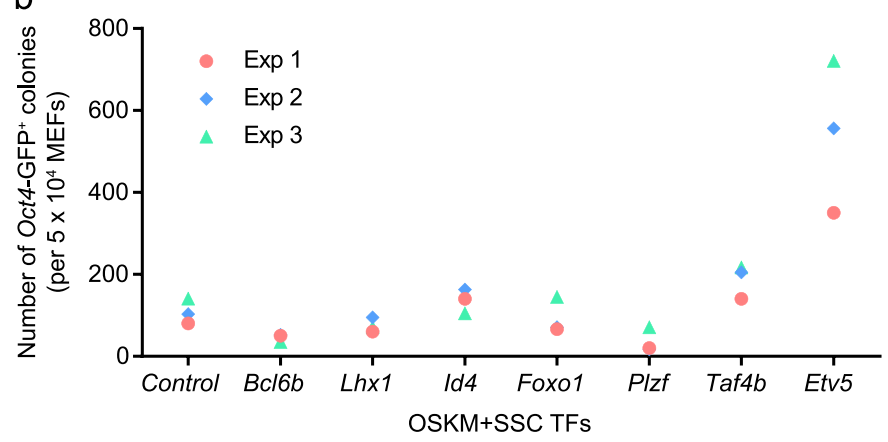

C

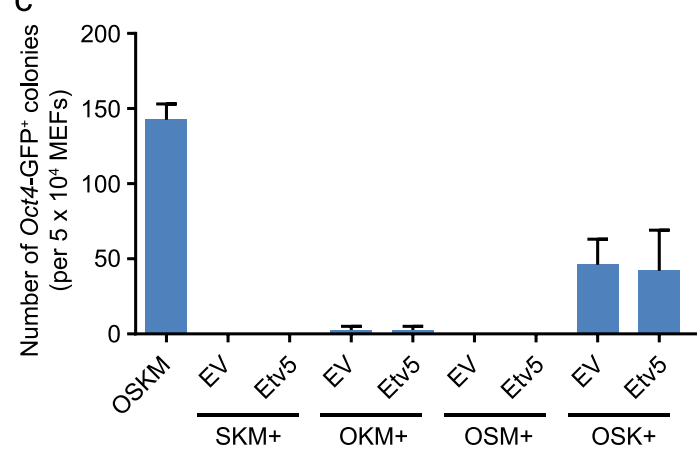

e

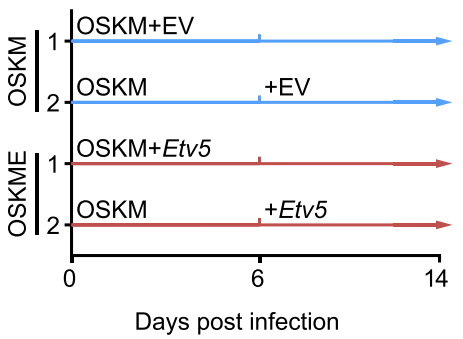

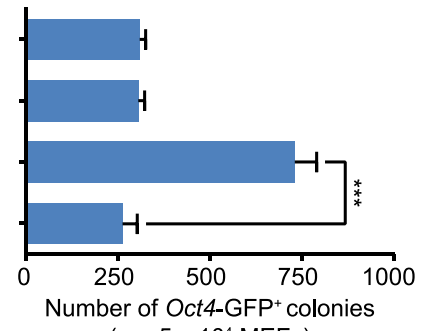

(per $5 \times 10^{4} \mathrm{MEFs}$ ) d

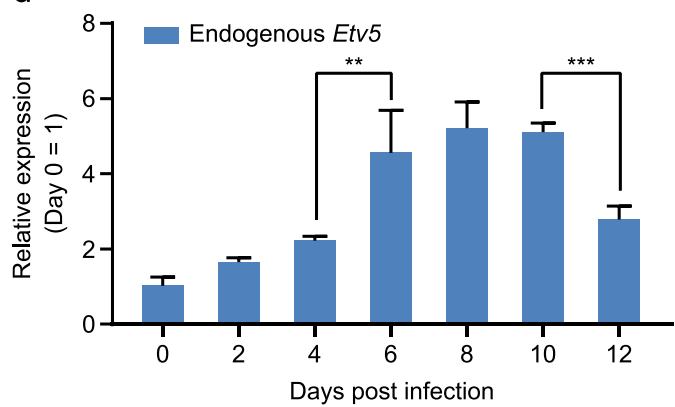

f

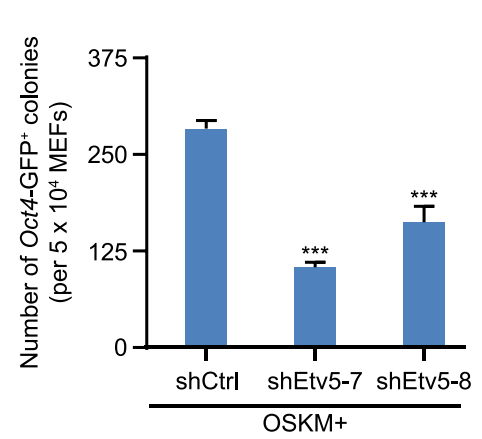

Fig. 1 Etv5 facilitates somatic cell reprogramming. a Diagram of iPSCs induction and germline-derived pluripotent stem cells (gPSC) induction. $\mathbf{b}$ Counts of Oct4-GFP ${ }^{+}$colonies at 16 days post infection (dpi) from combinations of SSCs TFs with Yamanaka factor OSKM. The results of three independent experiments (Exp) were shown. The combination of Yamanaka factors OSKM with pMXs empty vector (EV) was used as control. c Counts of Oct4-GFP ${ }^{+}$colonies at 16 dpi. OSKM was as positive control. The individual factor of OSMK was replaced by Etv5. The pMXs EV was used as vector control. d RT-qPCR analysis of endogenous Etv5 in OSKM mediated cell reprogramming. e Counts of Oct4-GFP ${ }^{+}$colonies at 14 dpi with two treatments. Introduction of Etv 5 at 0 and 6 dpi was compared. pMXs EV was used in parallel as vector control. f Counts of Oct4-GFP ${ }^{+}$colonies at 16 dpi from combinations of OSKM with Etv5 shRNA (shEtv5-7 and shEtv5-8). Nonsense shRNA (shCtrl) was used as vector control. Data are shown as mean \pm SD, Two-way ANOVA with Dunnett's multiple comparisons test was used for statistics analysis in $\mathbf{d}$ and $\mathbf{f}$; Two-way ANOVA with Sidak's multiple comparisons test was used for statistics analysis in $\mathbf{e} .{ }^{* *} P<0.01,{ }^{* * *} P<0.001(n=3)$

staining of infected cells (4 days post infection, 4 dpi) revealed that $\mathrm{CDH} 1$ positive cells were found more in OSKME when compared that with OSKM (Fig. 2a). Flow cytometry analysis of infected cells $(9 \mathrm{dpi})$ also confirmed that OSKME significantly increased CDH1 positive cells when compared that with OSKM. By contrast, endogenous Etv5 KD in OSKM combination decreased CDH1 positive cells (Fig. 2b). Furthermore, we investigated the time course expression of Etv5 and mesenchymal TFs (Zeb1, Zeb2, and Snail) at the early stage of reprogramming (Fig. 2c-f). We found that OSKME significantly decreased the expression levels of Snail, Zeb1, and Zeb2 when compared that with OSKM. However, Etv5 KD in OSKM only exclusively increased the expression level of Zeb1 (Fig. 2d). Together these results indicate that Etv5 could promote MET by decreasing Zeb1 expression. 
a
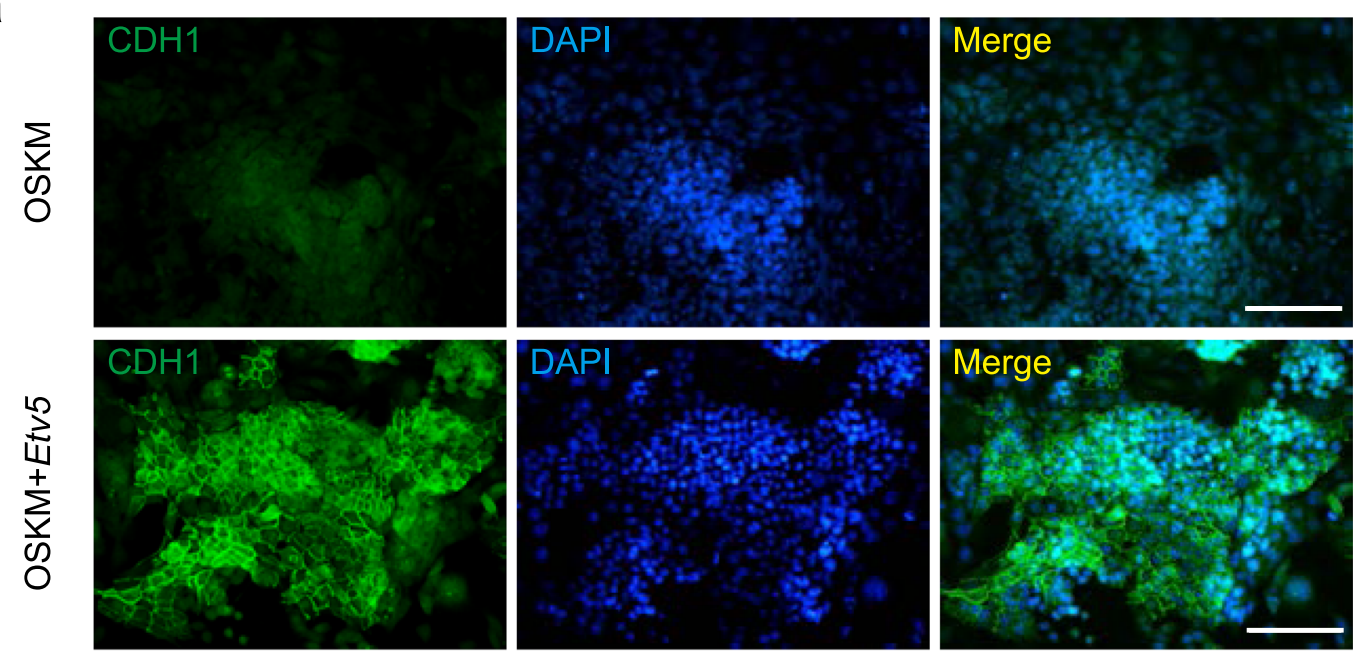

b

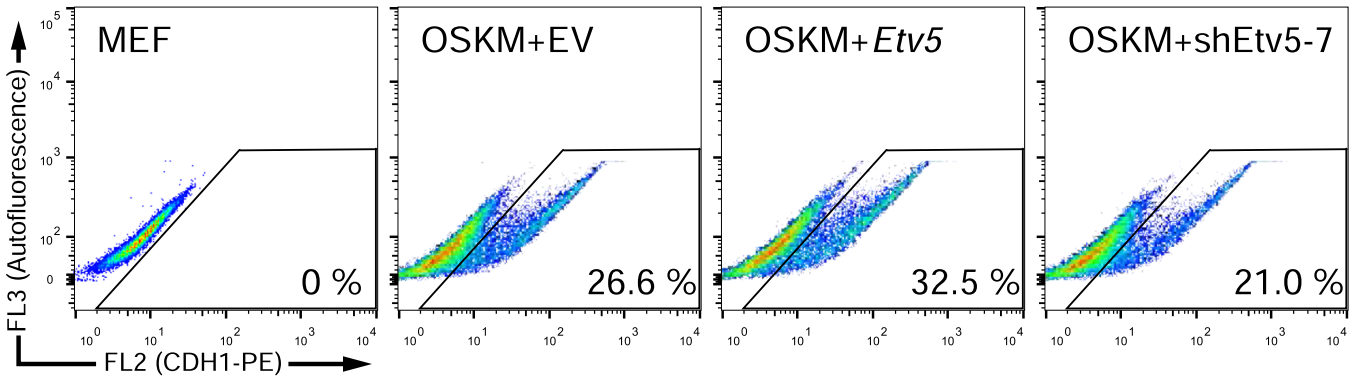

C

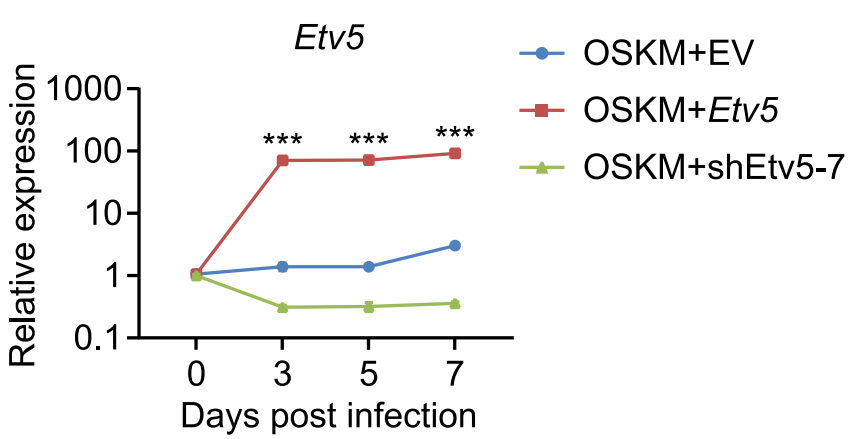

e

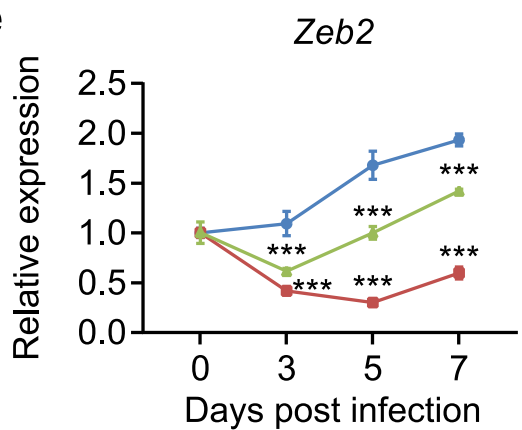

d

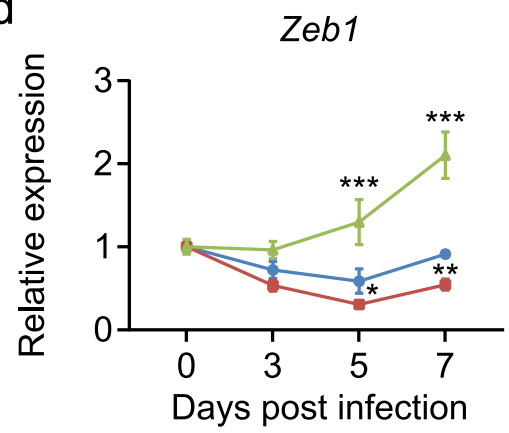

f

Snail

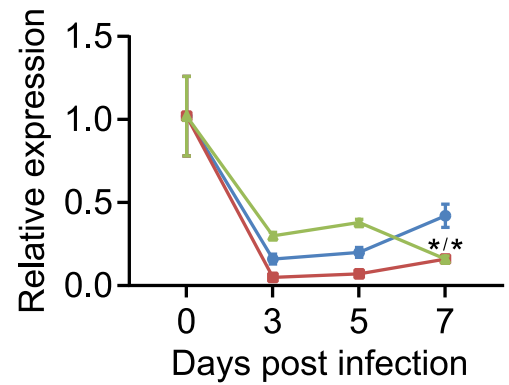

Fig. 2 (See legend on next page.) 
(see figure on previous page)

Fig. 2 Etv5 promotes somatic reprogramming by facilitating MET. a CDH1 immunofluorescence staining (green) of cells at $4 \mathrm{dpi}$ with OSKME and OSKM. Nucleus was stained with DAPI (blue). Scale bars, $100 \mu \mathrm{m}$. b Flow cytometric analysis of CDH1 positive cells at 9 dpi. The cells infected with OSKM + EV, OSKM + Etv5 and OSKM + shEtv5-7, respectively, were compared. MEFs were used as negative control. c-f RT-qPCR analysis of Etv5, Zeb1, Zeb2, and Snail at the early stage of reprogramming (Day 0-7). The cells infected with OSKM + EV, OSKM + Etv5 and OSKM + shEtv5-7, respectively, were compared. Two-way ANOVA with Dunnett's multiple comparisons test was used. Data are shown as mean \pm SD $(n=3)$. OSKM + EV was set as the control. ${ }^{*} P<0.05,{ }^{* *} P<0.01,{ }^{* * *} P<0.001$

\section{Etv5 promotes MET through Tet2-miR200s-Zeb1 axis}

Interactions between Zeb family (Zeb1 and Zeb2) and miR-200s family are suggested to regulate MET and epithelial-mesenchymal transition (EMT) in development, tumorigenesis, and reprogramming ${ }^{13,14}$. So we asked whether Zeb1 interacts with miR-200s family members during OSKME-mediated reprogramming. We first analyzed the complementation of 3' UTR of Zeb1 mRNA and miRNA-200 family members. Interestingly, there are five sites in 3' UTR of Zeb1 potentially targeted by cluster A of miRNA-200 family members (miR-200b,-200c, and -429) and three sites by cluster B of miRNA-200 family members (miR-200a and -141) (Fig. 3a). We then assessed whether overexpression of Etv 5 could increase expression levels of any miRNA-200 family members. Expectedly, all the miRNA-200 family members were found to be expressed with higher expression levels in OSKME combination when compared that with OSKM during the first seven days of reprogramming (Fig. 3b). However, Etv $5 \mathrm{KD}$ in OSKM combination only led to the decreased expression levels of miR-200a, miR-200b, and miR-429 (Fig. 3b).

Next, we asked whether Etv5 could directly regulate miR-200s family members during OSKME-mediated reprogramming. We analyzed the promoter regions (3.5 $\mathrm{kb}$ fragment upstream of transcription start site (TSS)) of miR-200s family members using online genomics comparative tool VISTA. Conserved noncoding sequences (CNS) were found across mammal species (Fig. 3c). However, we found no potential ETV5 binding sites located in these CNS regions. Alternatively, Tet proteins (TET1, TET2, and TET3) were proposed to directly regulate the differentially methylated region around TSS of miR-200s family ${ }^{15}$ (Fig. 3c). Therefore, we asked whether overexpression of Etv5 could increase the expression levels of Tet family members. Interestingly, only Tet 2 was found to be expressed with higher expression levels in OSKME combination when compared that with OSKM. Etv5 KD in OSKM also confirmed the exclusive contribution of Etv5 to Tet2 expression (Fig. 3d). Next, we performed luciferase assay to determine whether Etv5 could directly activate Tet 2 promoter or enhancer ${ }^{16}$. Two genomic fragments (Pro I and Pro II) with predicted Etv5 binding site showed promoter activity in NIH 3T3 cells, although the activities varied a lot (Supplementary
Fig. S3a, b). Then we co-transfected these constructs with Etv 5 overexpression vectors, but found no significant increase of luciferase expression for both Pro I and Pro II when compared with control (Supplementary Fig. S3c). Similar result was observed for fragment En I when comparing its enhancer activities between Etv5 and GFP overexpression conditions (Supplementary Fig. S3c). Together these results suggest that Etv 5 may regulate Tet 2 indirectly or involve of additional co-factors.

Collectively, the results above suggest that Etv5 can promote MET through Tet2-miR200s-Zeb1 axis (Fig. 3e).

\section{Etv5 KD decreases $5 \mathrm{hmC}$ level by downregulating Tet 2 in mESCs}

The beneficial effect of Etv 5 in somatic reprogramming intrigued us to investigate the roles of Etv 5 in mESCs maintenance. We firstly analyzed the expression levels of Etv5 among different cell types from BioGPS database, and found mESCs expressed the highest level of Etv5 when compared to somatic cells (Fig. 4a). We also analyzed the ChIP-seq and ChIP-chip data from ESCAPE database and constructed the interaction network between well-known pluripotent factors and Etv5 (Fig. 4b). Etv 5 is potentially regulated by 22 pluripotency relevant regulators, including POU5F1, SOX2, NANOG, ESRRB, SALL4, PRDM14, TCF3, MYC, NR0B1, YY1, ZFX, SMC1A, KDM5B, E2F1, E2F4, ZFP42, MAX, NIPBL, ASH2L, MED1, MED12, and ZIC3 (Fig. 4b). These analyses suggest Etv 5 to be an important member of pluripotency regulation network.

Then we infected J1 mESCs with Etv5 shRNA with puromycin resistance and tried to examine the phenotype of Etv5-KD mESCs. The efficiency of Etv5 knockdown was confirmed by RT-qPCR (Supplementary Fig. S4a). Compared to shEtv5-8, the shEtv5-7 showed the highest efficiency to downregulate Etv5. So we focused on shEtv57 and used it in the following assays. Although Etv5-KD colonies became looser in KSR + LIF medium, they could recover their compact morphology in $2 \mathrm{i}$ medium (Supplementary Fig. S4b).

To evaluate the effect of Etv5 KD on self-renewal of mESCs, we compared the proliferation and pluripotency genes expression between Etv5-KD and nonsense shRNA control colonies (abbreviated as shCtrl colonies hereafter). 
a

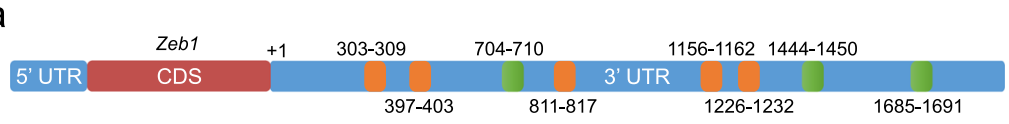

Predicted binding sites for cluster A Predicted binding sites for cluster B

Cluster A

Cluster B

miR-200b: uaauacugccugguaaugauga- miR-200a: uaacacugucugguaacgaugu miR-200c: uaauacugccggguaaugaugga miR-141: uaacacugucugguaaagaugg miR-429: uaauacugucugguaaugccgu

b
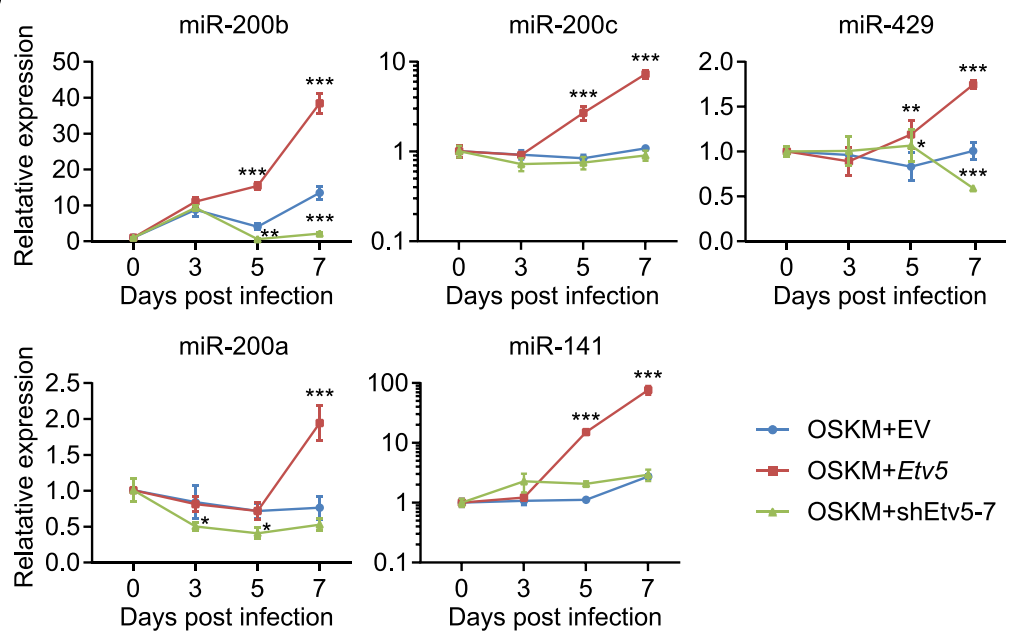

miR-141

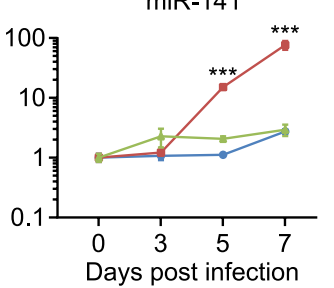

$\rightarrow$ OSKM+EV

- OSKM+Etv5

$\leadsto$ OSKM+shEtv5-7

C

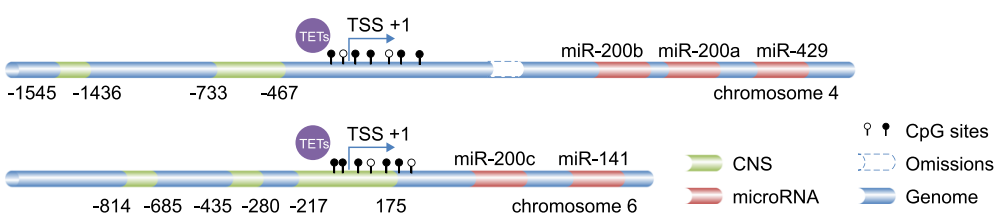

d
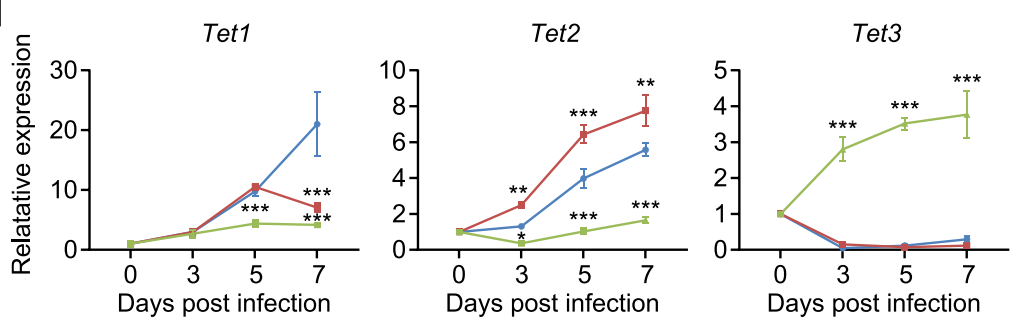

$\rightarrow$ OSKM+EV

$\rightarrow$ OSKM+Etv5

$\rightarrow$ OSKM+shEtv5-7

e

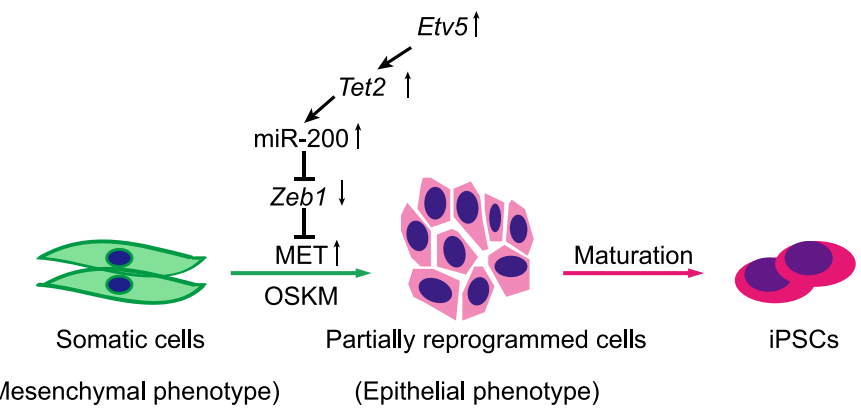

Fig. 3 (See legend on next page.) 


\begin{abstract}
(see figure on previous page)
Fig. 3 Etv5 promotes MET through Tet2-miR-200s-Zeb1 axis. a Predicted binding sites of miR-200 family located in 3' UTR of Zeb1. Five sites (orange) are supposed to be targeted by cluster A of miR-200 family (miR-200b,-200c and -429). Three sites (green) are supposed to be targeted by cluster B of miR-200 family (miR-200a, and -141). b RT-qPCR analysis of miR-200 family members during the early stage of reprogramming (Day 0-7). Cells infected with OSKM +EV, OSKM + Etv5 and OSKM + shEtv5-7, respectively, were compared. Two-way ANOVA with Dunnett's multiple comparisons test was used. Data are shown as mean $\pm \mathrm{SD}(n=3)$, OSKM + EV was set as the control, ${ }^{*} P<0.05$, ${ }^{* *} P<0.01$, ${ }^{* * *} P<0.001$. c The diagram of genomic structure of miR-200 family on chromosome 4 and chromosome 6 . The red regions represent miRNA clusters and green regions represent conserved noncoding sequence (CNS) across mammals. The differentially methylated region around transcription start site (TSS) of miR200s family is suggested to be demethylated by TET proteins (TET1, TET2, and TET3). $\mathbf{d}$ RT-qPCR analysis of Tet1, Tet2. and Tet3 during the early stage of reprogramming (Day 0-7). The cells infected with OSKM + EV, OSKM + Etv5 and OSKM + shEtv5-7, respectively, were compared. Two-way ANOVA with Dunnett's multiple comparisons test was used. Data are shown as mean $\pm \mathrm{SD}(n=3)$, OSKM + EV was set as the control, ${ }^{*} P<0.05,{ }^{* *} P<0.01,{ }^{* * *} P$ $<0.001$. e The working model of Etv5 in somatic reprogramming. Overexpression ( $\uparrow$ ) of Etv 5 with Yamanaka factor (OSKM) can facilitate MET through Tet2-miR-200s-Zeb1 axis at the early stage of cell reprogramming
\end{abstract}

Compared to shCtrl colonies, the proliferation rate of Etv5-KD colonies was lowered (Fig. 4c). However, there were no obvious differences between shCtrl colonies and Etv5-KD colonies on alkaline phosphatase staining and immunofluorescence of OCT4, SOX2, and SSEA-1 (Supplementary Fig. S4c). These results indicate that Etv5 is dispensable for pluripotency maintenance although Etv5-KD colonies compromise their proliferation.

We next asked whether Etv5 could positively regulate Tet2 in mESCs as seen in somatic reprogramming. RTqPCR and Western blotting both showed that Tet 2 was significantly downregulated in Etv5-KD colonies (Fig. 4d, e). Furthermore, we did dot blot experiment for $5 \mathrm{hmC}$ and found the genome $5 \mathrm{hmC}$ level was expectedly decreased as Etv5 was downregulated (Fig. 4f). The downregulation of Tet 2 and genomic $5 \mathrm{hmC}$ level in Etv5KD mESCs was rescued by re-introducing Etv 5 (Fig. $4 \mathrm{~d}$-f). Collectively, these results indicate that Etv 5 can positively regulate Tet 2 expression and thus influence the genomic $5 \mathrm{hmC}$ level in mESCs.

\section{Transcriptome changes caused by Etv5 KD in mESCs}

The paradoxical findings of Etv 5 in mESCs maintenance prompted us to hypothesize that Etv 5 may function mainly in mESCs differentiation. We then profiled the genes upregulated and downregulated in Etv5-KD mESCs by RNA-seq. Of note, 1049 genes were found to be significantly influenced by Etv $5 \mathrm{KD}\left(\log _{2} \mathrm{FC}>1, \mathrm{FDR}<0.05\right)$ (Fig. 5a, left). Gene ontology (GO) analysis revealed that these differentially expressed genes showed high enrichment in GO terms like angiogenesis, MAPK signaling pathway, urogenital system development and sensory organ (eye) morphogenesis (Fig. 5b). To further explore the potential biological effects caused by Etv 5 through Tet2, We carried out paralleled analysis of RNA-seq data from Tet2-KD mESCs and their corresponding shCtrl $\mathrm{mESCs}^{17}$. There were five original pairs (Tet2-KD versus shCtrl) of RNA-Seq replicates and principal component analysis (PCA) revealed poor consistency of pair 3 and pair 5 (Supplementary Fig. S5a). Therefore, we used the filtered pairs (1,2, and 4) for the subsequent analysis (Supplementary Fig. S5b). Interestingly, only 477 genes were found to be differentially expressed between Tet2$\mathrm{KD}$ and shCtrl mESCs $\left(\log _{2} \mathrm{FC}>1, \mathrm{FDR}<0.05\right)$ (Fig. 5a, right) and these genes were highly enriched in biological process like gland morphogenesis (predominantly liver and prostate), wound healing, blood coagulation, and small molecule biosynthetic process (Fig. 5b and Supplementary Fig. S5c-e). Of note, there is broad overlap of Tet $2 \mathrm{GO}$ terms with that of Etv5, suggesting that multiple biological effects of Etv 5 may be mediated through Tet2.

Furthermore, we asked what genes were commonly regulated by both Etv 5 and Tet 2 . Venn analysis revealed that 12 genes were commonly upregulated and 15 genes were commonly downregulated in both Etv5-KD and Tet2-KD mESCs (Fig. 5c). Heatmap analysis of these commonly changed genes in Etv5-KD and Tet2-KD mESCs were shown in Fig. 5d and Fig. 5e, respectively.

Briefly, the transcriptome analysis indicates that Etv5 and Tet 2 have overlapped functions in regulating mESCs differentiation. Since Etv5 could function upstream to positively regulate Tet 2 as demonstrated before, these common differentially expressed genes are supposed to be downstream targets of Etv5-Tet2 regulation axis.

\section{Etv5 orchestrates the specification of primitive endoderm and epiblast during $\mathrm{mESCs}$ differentiation in vitro}

Among the 15 commonly downregulated genes, there were two genes (Gata6 and Pdgfra) which were proposed to regulate primitive endoderm differentiation in the second fate determination of late blastocyst ${ }^{18}$. Therefore, we speculated that Etv5-Tet2 regulation axis may facilitate primitive endoderm differentiation by maintaining Gata6 or Pdgfra at a poised state in mESCs. Gata6 is a master TF of primitive endoderm differentiation ${ }^{18}$, so we focused on it and did RT-qPCR validation. As Etv5 was knocked down in mESCs, both Tet 2 and Gata6 were similarly 
a

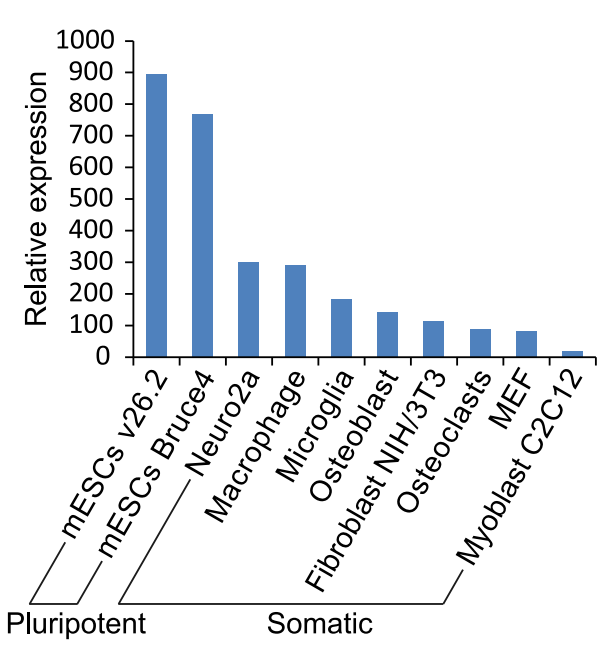

C

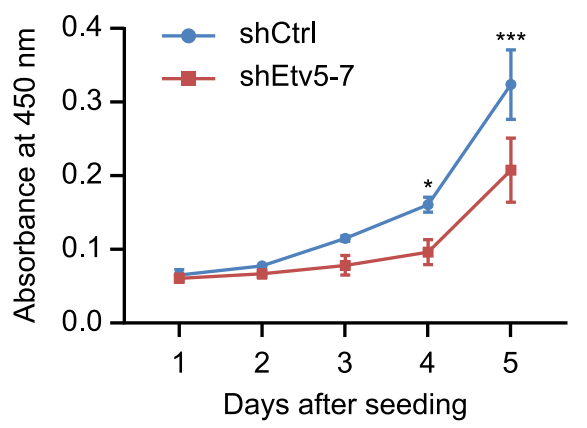

e

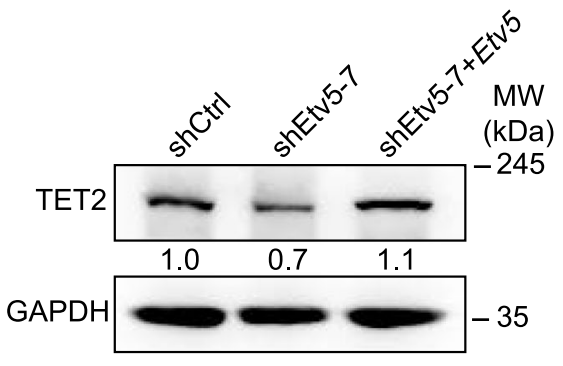

b

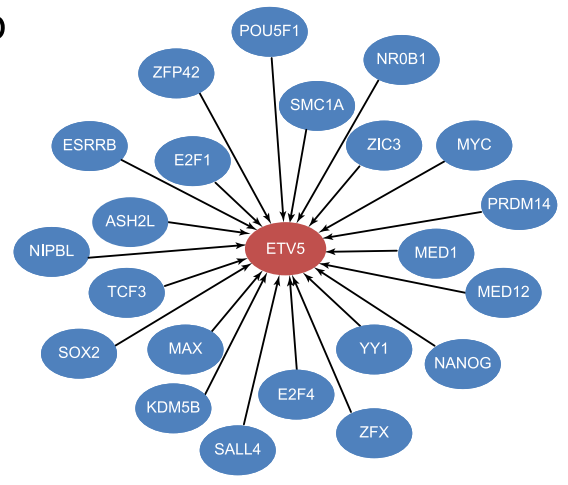

d

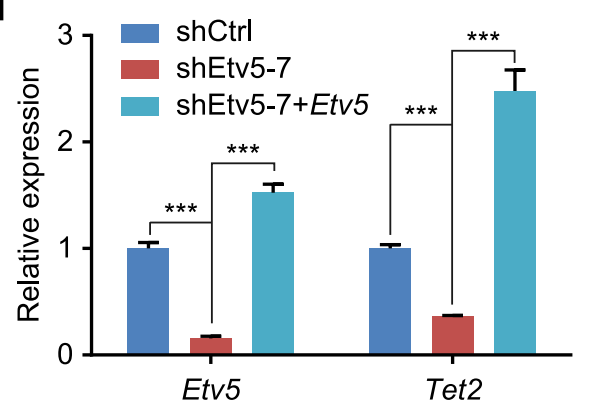

$f$

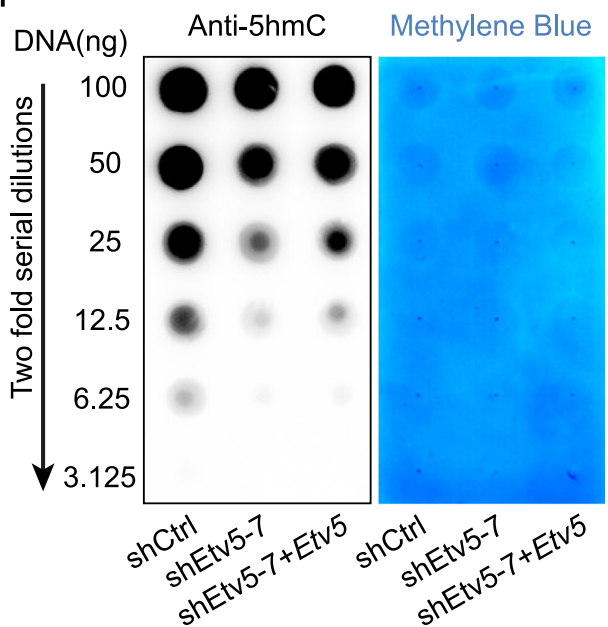

Fig. 4 Etv5 positively regulates Tet2 but is dispensable for mESCs maintenance. a Comparison of Etv5 expression levels between mESC lines and somatic cell lines. The relative expression was based on the microarray data from BioGPS database. $\mathbf{b}$ The interactions between pluripotency relevant regulators and Etv5. ChIP-seq and ChIP-chip data with Etv5 as target were extracted from ESCAPE database and used for drawing these interactions. c Growth curve of J1 mESCs stably infected with shCtrl and Etv5 shRNA (shEtv5-7). d RT-qPCR analysis of Etv5 and Tet2 in mESCs stably infected with shCtrl, Etv5 shRNA (shEtv5-7), and shEtv5-7 plus lentiviral Etv5. Data are shown as mean \pm SD $(n=3)$. ${ }^{*} P<0.05$, ${ }^{* * *} P<0.001$. Two-way ANOVA with Sidak's multiple comparisons test was used for $\mathbf{c}$. One-way ANOVA with Dunnett's multiple comparisons test for $\mathbf{d}$. e Western blotting of TET2 in mESCs stably infected with shCtrl, shEtv5-7, and shEtv5-7 + Etv5. GAPDH was used as internal control. The relative quantification is also shown. f Dot blot of global 5hmC in mESCs stably infected with shCtrl, shEtv5-7, and shEtv5-7 plus lentiviral Etv5. The blotting result of serially diluted genomic DNA (100-3.125 ng) was shown (left panel). The same membrane stained with methylene blue as DNA loading control was also presented (right panel)

downregulated (Fig. 6a), which was consistent to the results as observed in RNA-seq (Fig. 5d,e). Even more, we demonstrated that the downregulation of Tet2 and Gata6 in Etv5-KD mESCs was recovered as Etv5 was reintroduced (Fig. 6a). Together these findings indicate that Etv 5 may positively function upstream of Tet 2 which 


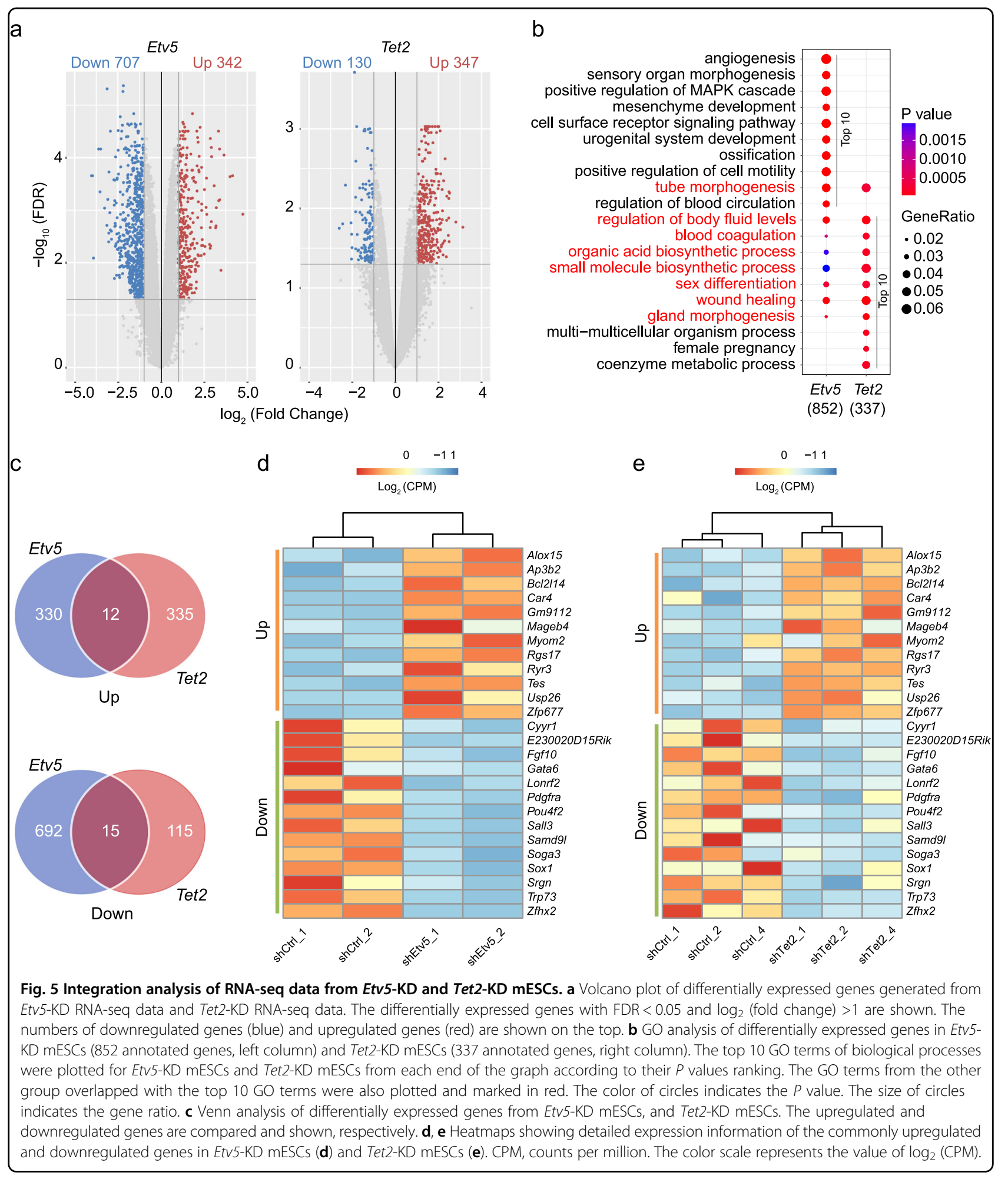

further positively regulates Gata6 at a poised state in undifferentiated mESCs.

To examine whether the relationship of positive regulation among Etv5, Tet2, and Gata6 also exists as mESCs differentiated, we investigated the time course expression of them during EB differentiation (Fig. 6b). Interestingly, we found that there was a fluctuation of Etv5 expression during an eight-day EB differentiation (Fig. 6b, left). According to the fluctuation of Etv5 expression level, we divided the eight days of EB differentiation into three 


\section{a}

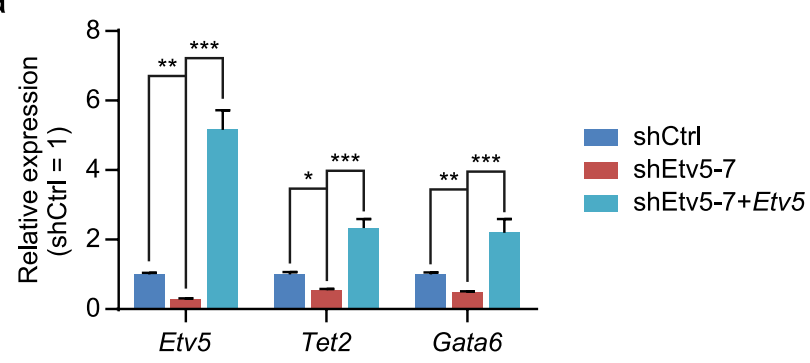

b

$\rightarrow$ shCtrl $\rightarrow$ shEtv5-7
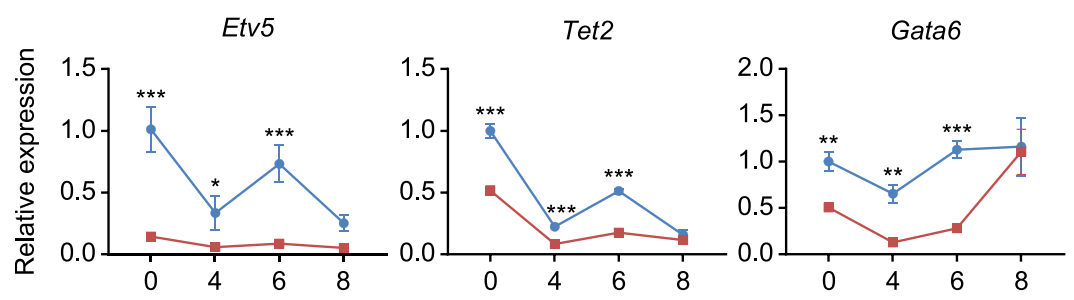

C
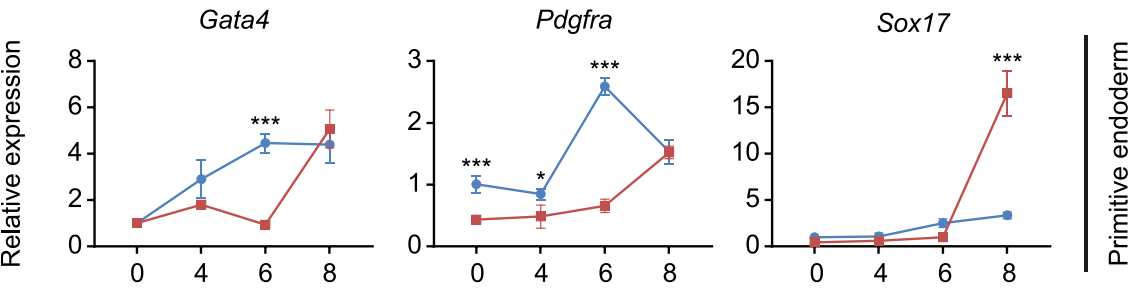

d

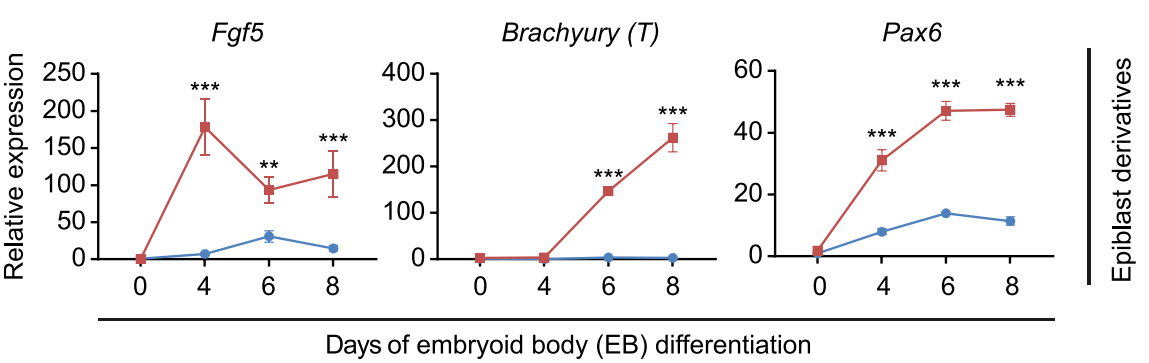

e

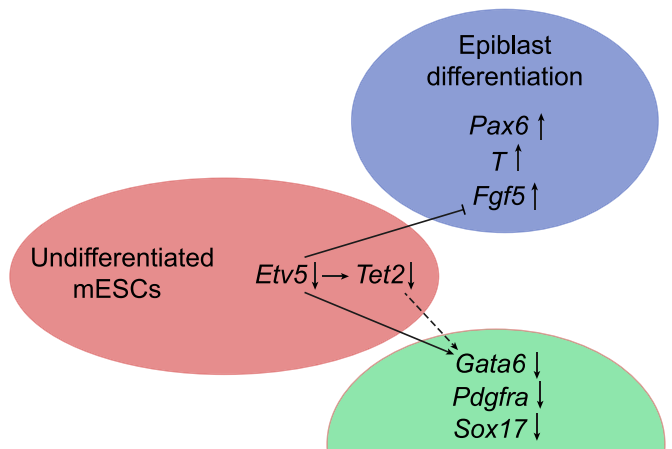

Primitive endoderm

differentiation

Fig. 6 (See legend on next page.) 


\begin{abstract}
(see figure on previous page)
Fig. 6 Etv 5 orchestrates the specification of primitive endoderm and epiblast during mESCs differentiation in vitro. a RT- $q P C R$ verification of the positive regulation relationship among Etv5, Tet2, and Gata6 in undifferentiated mESCs. The mESCs stably infected with nonsense shCtrl, shEtv5-7, and shEtv5-7 plus lentiviral Etv5 are compared. One-way ANOVA with Dunnett's multiple comparisons test was used. Data are shown as mean \pm SD $(n=3) .{ }^{*} P<0.05,{ }^{* *} P<0.01,{ }^{* * *} P<0.001$. b RT-qPCR analysis of Etv5, Tet2, and Gata6 during mESCs differentiation in vitro. c RT-qPCR analysis of of primitive endoderm marker genes (Gata4, Pdgfra, and Sox17) during mESCs differentiation in vitro. d RT-qPCR analysis of epiblast marker gene and its derivatives marker genes during mESCs differentiation in vitro. The expression of Fgf5 (epiblast), $T$ (mesoendoderm) and Pax6 (ectoderm) was observed. The mESCs stably infected with shCtrl and shEtv5-7 were used for EB differentiation and compared in $\mathbf{b}-\mathbf{d}$. Data are shown as mean \pm SD $(n=3)$. Two-way ANOVA with Sidak's multiple comparisons test was used in $\mathbf{b}-\mathbf{d} .{ }^{*} P<0.05,{ }^{* *} P<0.01,{ }^{* * *} P<0.001$. e The working model of Etv5 in mESCs differentiation. Etv5 knockdown $(\downarrow)$ can delay the primitive endoderm specification by downregulating Gata6. In parallel, Etv5 knockdown can promote specification of epiblast and its derivative mesoendoderm and ectoderm
\end{abstract}

phases: phase 1 (Day 0-4), the relative expression level (REL) of Etv 5 dropped from 1 to $\sim 0.3$; phase 2 (Day 4-6), the REL of Etv 5 upregulated from $\sim 0.3$ to $\sim 0.7$; phase 3 (Day 6-8), the REL of Etv5 then dropped from $\sim 0.7$ to $\sim 0.2$. Surprisingly, the fluctuation trend of Tet 2 and Gata6 was highly matched to the fluctuation expression of Etv 5 from phase 1 to 3 (Fig. 6b, middle and right). The REL of Etv5 in Etv5-KD mESCs showed similar fluctuation pattern, but at quite low level during the whole process of EB differentiation in parallel (Fig. 6b, left). Expectedly, the REL of Tet2 and Gata6 in Etv5-KD EB was significantly lower than that in shCtrl EB at the end of phase 1 and phase 2 (Fig. 6b, middle and right). Due to the similarly low level of Etv 5 between shCtrl EB and Etv5-KD $\mathrm{EB}$ at the end of phase 3, there was no significant difference on the REL of Tet 2 and Gata6 at this time point (Fig. 6b). Collectively, these results suggest that the relationship of positive regulation among Etv5, Tet2, and Gata6 faithfully exists during mESCs differentiation.

These findings above intrigued us to investigate whether Etv5 KD can lead to a delayed primitive endoderm differentiation. Although no obvious morphology difference was found between shCtrl EB and Etv5-KD EB (Fig. S6), the REL of primitive endoderm marker genes (Gata4 and Pdgfra) in Etv5-KD EB was significantly lower than that in shCtrl EB at the end of phase 1 and phase 2 (Fig. $6 \mathrm{c}$, left and middle). Unexpectedly, primitive endoderm marker gene Sox17 showed slight expression difference between shCtrl EB and Etv5-KD EB at the end of phase 1 and phase 2 (Fig. 6c, right). Interestingly, Sox 17 expression in Etv5-KD EB increased dramatically at the end of phase 3 and exceeded its expression in shCtrl EB (Fig. 6c, right). Summarily, these EB results indicate that primitive endoderm specification is supposed to be positively regulated by Etv5-Tet2-Gata6 axis during mESCs differentiation in vitro.

Binary segregation of inner cell mass into primitive endoderm and epiblast is regarded as the second fate decision of preimplantation embryo ${ }^{19}$. So we further asked whether Etv5 can negatively regulate the epiblast differentiation. We observed the expression trend of Fgf5, one master gene of epiblast, during EB differentiation. Surprisingly, the REL of Fgf5 in Etv5-KD EB was significantly higher than that in shCtrl EB from phase 1 to 3 (Fig. 6d, left). As the preimplantation embryo further progresses, the epiblast is believed to specialize into mesoendoderm and ectoderm ${ }^{20}$. So we tested whether the expression of mesoendoderm marker gene Brachyury $(T)$ and ectoderm marker gene (Pax6) increased as Fgf5 did. Expectedly, the REL of $T$ and Pax6 in Etv5-KD EB was both significantly higher than that in shCtrl EB during the whole process of differentiation (Fig. 6d, middle and right). Briefly, the specification of epiblast and its derivatives is supposed to be negatively regulated by Etv 5 during mESCs differentiation in vitro.

Taken together, these EB differentiation results indicate that Etv5 mainly functions to orchestrate the specification of primitive endoderm and epiblast during mESCs differentiation in vitro (Fig. 6e).

\section{Discussion}

In this study, we demonstrated that Etv 5 could promote MET at the early stage of reprogramming through Tet2miR200-Zeb1 regulation axis, which further increased the final efficiency of iPSCs induction (Fig. 3e). In addition, Etv5 KD in mESCs could decrease the genomic $5 \mathrm{hmC}$ level by downregulating Tet2. Moreover, Etv 5 could orchestrate the specification of primitive endoderm and epiblast during mESCs differentiation in vitro (Fig. 6e). Our findings indicate the novel mechanisms of Etv5 in somatic reprogramming, mESCs maintenance, and differentiation.

Etv 5 was found to be upregulated in the early stage of OSKM-mediated reprogramming ${ }^{7}$. Our study confirmed this finding and further revealed that Etv 5 could promote reprogramming efficiency when combined with OSKM. By contrast, Etv 5 could not increase reprogramming efficiency when combined with OSK (Fig. 1c). These findings suggest that cooperation between Etv5 and c-Myc may be essentially required for Etv 5 to promote reprogramming. Interconnectivity analysis of pluripotent TFs in human ESCs indicates ETV5 as a co-regulator of 
c-MYC ${ }^{21}$. Further study of interactions between $E t v 5$ and $c-M y c$ may provide insights into the mechanism of OSKME-mediated reprogramming.

The process of reprogramming MEFs into iPSCs is generally divided into early, middle, and late stage ${ }^{12}$. One of the critical features in early stage of reprograming is MET, which is believed to be orchestrated by TFs, epigenetic modifiers, and signaling pathways ${ }^{22,23}$. This is exemplified by the finding that Tet proteins and TDG glycosylase can mediate DNA demethylation and reactivation of miRNAs critical for MET in OSKM-mediated reprogramming ${ }^{15}$. In OSKME-mediated reprogramming, Etv5 could contribute exclusively the improvement of MET by Tet2-miR200s-Zeb1 regulation axis. This reflects a cooperation of transcriptional regulation and posttranscriptional regulation during the process of MET. We also noted that Etv5 KD in OSKM could not completely block MET, which indicated additional regulation axis must contribute the MET at the early stage of reprogramming. This speculation is consistent with the observation that Oct4/Sox2 can induce MET by miR-200Zeb2 pathway ${ }^{14}$. In addition, BMPs molecules in the serum-containing mESCs medium may also facilitate MET in OSKME-mediated reprogramming ${ }^{24}$.

Etv 5 was once predicted as an important member of mESCs pluripotency network ${ }^{25}$. ChIP-seq and ChIP-chip analysis of classical pluripotency relevant TFs also support the view that Etv5 is integrated into the regulatory network of pluripotency (Fig. 4b). Ironically, we observed no dramatic changes on colony morphology and expression level of classical pluripotency TFs in Etv5-KD mESCs. This phenotype may be partially explained by the functional redundancy between Etv 4 and Etv 5 in $\mathrm{mESCs}^{6}$.

By contrast, Etv 5 orchestrated the specification of primitive endoderm and epiblast during mESCs differentiation in vitro. These findings provide complementary evidences to understand the roles of Etv5 in fine tuning cell fate determination of primitive endoderm and epiblast in vivo ${ }^{26}$. Interestingly, Etv5 is a direct target of Gata6 during primitive endoderm differentiation ${ }^{27}$. Therefore, it is likely that the primitive endoderm specification is controlled by a negative feedback between Etv 5 and Gata6. Whether there is a direct interaction of Tet2 and Gata6 remains to be examined in the future.

Collectively, our findings in this study provide insights into understanding the mechanisms of Etv 5 in somatic reprogramming, mESCs maintenance and differentiation in vitro.

\section{Materials and methods}

\section{Cell culture}

Mouse embryonic fibroblasts (MEFs) were derived from 13.5 d.p.c mouse embryos by crossing male ICR mice to female OG2 mice (B6;CBA-Tg(Pou5f1-EGFP) 2Mnn/J).
Feeder cells were prepared by treating MEFs with $10 \mu \mathrm{g}$ $\mathrm{ml}^{-1}$ mitomycin C (Sigma). MEFs were maintained in high glucose DMEM (Hyclone) supplemented with 15\% FBS (Gibco) and $0.1 \mathrm{mM}$ non-essential amino acids (Gibco). HEK293T and Plat-E cells were maintained in DMEM supplemented with $10 \%$ FBS. For routine culture, iPSCs and J1 (129/Sv) mESCs were maintained on feeder layers in standard mESCs medium. The standard mESCs medium was composed of high glucose DMEM, 15\% FBS, $100 \mu \mathrm{M}$ non-essential amino acids, $1 \mathrm{mM}$ L-glutamine (Gibco), $100 \mu \mathrm{M} \beta$-mercaptoethanol (Gibco), $100 \mu \mathrm{g} \mathrm{ml}^{-1}$ Vitamin C (Sigma) and 1000 units $\mathrm{ml}^{-1}$ leukaemia inhibitory factor (LIF) (Millipore). For gene knockdown and differentiation experiments, J1 mESCs were maintained in feeder-free conditions on $0.1 \%$ gelatin-coated tissue culture plates with KSR based mESCs medium which was modified from standard mESCs medium. The component of $15 \%$ FBS in standard mESCs medium was replaced by $12 \%$ knockout replacement serum (KSR) (Gibco) plus 3\% FBS. To compare the pluripotent gene expression of iPSCs under different culture conditions, pluripotent cells maintained in standard mESCs medium and $2 \mathrm{i}$ medium were used. The $2 \mathrm{i}$ medium consists of N2B27 medium, $100 \mu \mathrm{M} \beta$-mercaptoethanol, $1 \mu \mathrm{M}$ PD0325901 (StemRD), $3 \mu \mathrm{M}$ CHIR99021 (StemRD) and $1000 \mathrm{U} \mathrm{ml}^{-1}$ LIF. The medium described above was changed daily and all the cells lines were routinely tested to keep mycoplasma free.

\section{Plasmid construction}

For the package of retrovirus carrying SSCs specific TFs, coding sequences (CDS) of candidate TFs were amplified from cDNA library of mouse testis and cloned into the pMXs vector. The lentiviral vector pTrip-CAGG-Etv5IRES-Neo was constructed by replacing puro cassette in pTrip-CAGG-MCS-IRES-puro plasmid with NeoR gene first and followed by insertion of Etv5 CDS. pSicoRshEtv5-7-puro, pSicoR-shEtv5-8-puro, and control vector pSicoR-shCtrl-puro are kind gifts from Dr. Marius Wernig and designed to target the 3' UTR of Etv5. The specificity of these Etv 5 shRNAs vectors had been validated in the publication by Lujan $\mathrm{E}$ et al. ${ }^{7}$. For reporter plasmid construction, putative promoter fragments were amplified from MEFs genomic DNA using Phanta Max SuperFidelity DNA Polymerase (Vazyme) and subcloned into the multiple cloning sites of pGL3-Basic vector (Promega). Putative enhancer fragment were subcloned into the pGL3-Promoter vector (Promega) containing a minimal simian virus40 (SV40) promoter at the MluI/ XhoI sites. All the plasmids described above were verified by sequencing and primers involved are listed in Table S1.

\section{Dual-luciferase reporter assay}

One day before the transfection, NIH 3T3 cells were seeded at $2.5 \times 10^{4}$ cells per well of a 24 -well plate. All 
pGL3 constructs were co-transfected with pRL-TK and pMXs-Etv5 using lipofectamine 2000 (Invitrogen). Cells were harvested 2 days after transfection for measurement of luciferase activities by using Dualluciferase reporter assay system (Promega). Three independent transfections in triplicate were performed for the luciferase assay.

\section{Mouse iPSCs induction}

Mouse iPSCs were generated with retroviruses as described previously ${ }^{1}$ with some modifications. Briefly, Plat-E cells were seeded at a density of $6 \times 10^{6}$ cells per $100-\mathrm{mm}$ culture dish one day before the transfection. The pMXs-based retroviral vectors $(20 \mu \mathrm{g})$ were transfected into Plat-E cells using Calcium Phosphate Cell Transfection Kit (Beyotime Biotechnology) according to the manufacturer's instruction. The medium was changed 12 $\mathrm{h}$ later. Virus-containing supernatants were collected twice at $48 \mathrm{~h}$ and $72 \mathrm{~h}$ post transfection, and filtered through $0.45 \mu \mathrm{M}$ PVDF filter (Millipore). Polybrene (Sigma) was added to the supernatant at a final concentration of $8 \mu \mathrm{g} \mathrm{ml}^{-1}$ before the cocktail of TFs retroviruses was added to the MEFs. The MEFs within three passages were seeded at $3.5 \times 10^{4}$ cells per well in a 6-well plate the day before transduction, and incubated with the retrovirus supernatant for $12 \mathrm{~h}$. After two rounds of infection, the medium was changed to standard mESCs medium and changed daily. Oct4-GFP ${ }^{+}$colonies were counted on indicated days, after which colonies were picked up for expansion. For induction of iPSCs from Etv5-shRNA infected MEFs, puromycin $\left(2 \mu \mathrm{g} \mathrm{ml}^{-1}\right)$ was added to the standard mESCs medium after final round of infection and removed 3 days later.

\section{Immunofluorescence staining}

The mESCs colonies were fixed with 4\% PFA for $20 \mathrm{~min}$ at room temperature, washed three times with PBS and permeabilized with $0.1 \%$ Triton $\mathrm{X}-100$ at room temperature for $20 \mathrm{~min}$. After three washes with PBS, the cells were blocked with 5\% FBS for one hour at room temperature and incubated with diluted primary antibody overnight. The next day, the cells were washed three times with PBS and incubated with fluorophore-conjugated secondary antibody for one hour at room temperature and followed by DAPI staining. The images were taken under the fluorescence microscope (EVOS FL, Thermo Scientific). The following antibodies were employed in this study: anti-SSEA1 (1:100, 4744s, CST), anti-Nanog (1:300, ab80892, Abcam), anti-Oct-3/4 (1:200, sc-5279, Santa Cruz), anti-E-cadherin/CDH1 (1:1000, sc8426, Santa Cruz), anti-Neuronal Class III $\beta$-Tubulin (1:1000, MMS-435P, Covance), anti-DESMIN (1:500, MAB3430, Millipore), anti-AFP (1:50, MAB1368, R\&D System).

\section{Transgenes integration and exogenous transgenes silencing}

For transgenes integration detection, genomic DNA of mouse iPSCs was extracted using TIANamp Genomic DNA Kit (TIANGEN). For exogenous transgenes silencing detection, cDNA library was prepared as described below. PCR reactions were conducted using Taq DNA Polymerase (Fermentas) on S1000 Thermal Cyclers (BioRad). Primers employed in this section were listed in Table S1.

\section{Reverse transcription PCR and real-time PCR}

Total RNA was extracted using RNAiso Plus (TaKaRa) according to the manufacturer's instruction. First strand cDNA synthesis was conducted using RevertAid First Strand cDNA Synthesis Kit (Thermo Scientific), during which the genomic DNA was removed with DNase I (Thermo Scientific) treatment. For the quantification of mRNA expression, a mixture of oligo (dT)18 primer and random hexamer primer was employed in the reverse transcription reaction and quantitative real-time PCR was performed using TransStart ${ }^{\circ}$ Tip Green qPCR SuperMix (TansGen Biotech) on StepOnePlus Real-Time PCR System (Applied Biosystems). For the quantification of microRNA expression, total RNA was reverse transcribed with pre-designed gene-specific Bulge-LoopTM RT primers (Ribobio) and quantitative real-time PCR was performed using Bulge-LoopTM miRNA qPCR Primer Sets (Ribobio) following the vendor's manual. The primers for qPCR analysis of genes involved in this study can be found in Table S1.

\section{Bisulfite sequencing}

The promoter methylation of Oct4 and Nanog was analyzed for J1 mESCs, OSKME-4, OSKME-5 iPSCs, and MEFs. Briefly, genomic DNA $(1 \mu \mathrm{g})$ is subjected to sodium bisulphite conversion using EpiTect Bisulfite Kit (Qiagen). Purified genomic fragments were amplified using AceTaq DNA Polymerase (Vazyme) through nested or seminested PCR reactions. The PCR products were then cloned into pGEM-T Easy vector (Promega), after which individual transformants were screened by colony PCR and positive colonies were sequenced. The sequencing results were analyzed with BiQ Analyzer . PCR primers used for bisulfite sequencing can be found in Table S1.

\section{Western blotting}

Cells were washed cold PBS and lysed on ice for $15 \mathrm{~min}$ with RIPA buffer (Vazyme) supplemented with protease inhibitor cocktail (Roche), after which the cell lysate was collected and centrifuged to eliminate the cell debris. The supernatant mixed with loading buffer was boiled at $100^{\circ}$ $\mathrm{C}$ for $5 \mathrm{~min}$ and subjected to SDS-PAGE separation. The protein was then transferred to PVDF membrane 
(Invitrogen) and blocked with 5\% nonfat dry milk (BD Biosciences) for $1 \mathrm{~h}$ at room temperature. The blot membrane was incubated with primary antibody overnight at $4{ }^{\circ} \mathrm{C}$, washed three times with TBST buffer and incubated with HRP-conjugated secondary antibody for 1 $h$ at room temperature. Signals were detected using Highsig ECL Western Blotting Substrate (Tanon). The antibodies used for immunoblotting were anti-TET2 (1:1500, 21207-1-AP, Proteintech) and anti-GAPDH (1:5000, KM9002, SUNGENE BIOTECH).

\section{Dot blot}

Purified genomic DNA was denatured in $100 \mathrm{mM}$ $\mathrm{NaOH}, 10 \mathrm{mM}$ EDTA for $10 \mathrm{~min}$ at $95^{\circ} \mathrm{C}$ and neutralized with $1 \mathrm{M} \mathrm{NH}_{4} \mathrm{OAc}$ on ice for $5 \mathrm{~min}$, after which they were diluted to a final concentration of $25 \mathrm{ng} \mathrm{\mu l}^{-1}$ and then serially diluted. Equal volume of samples was spotted onto the pre-balanced nitrocellulose membrane. The membrane was incubated at $80^{\circ} \mathrm{C}$ for $5 \mathrm{~min}$ and subjected to UV cross-link at $120,000 \mu \mathrm{J} \mathrm{cm}^{-2}$. Then the membrane was blocked with $5 \%$ nonfat dry milk for $1 \mathrm{~h}$ at room temperature, incubated with anti-5hmC antibody (1:6000, 39791, Active Motif) overnight at $4{ }^{\circ} \mathrm{C}$, and washed three times with TBST buffer. The membrane was next incubated with HRP-conjugated secondary antibody for $1 \mathrm{~h}$ at room temperature, and the signal was detected with ECL reagents. The same membrane was finally stained with $0.02 \%$ methylene blue for $10 \mathrm{~min}$ in $0.3 \mathrm{M}$ sodium acetate (pH 5.2) to ensure equal spotting of the total genomic DNA on the membrane.

\section{EB differentiation}

For mESCs or iPSCs cultured on feeder layers, colonies were trypsinized into single cells and transferred onto gelatin coated culture dish in standard mESCs medium, the cells were incubated at $37^{\circ} \mathrm{C}$ for $45 \mathrm{~min}$ to eliminate the feeder cells, after which the floating cells were collected by centrifugation. Then $1.2 \times 10^{6}$ cells were suspended with differentiation medium (standard mESCs medium without LIF) and transferred to $60 \mathrm{~mm}$ petri dish for further differentiation. The differentiation medium for EB culture was changed every 2 days. EB samples were collected on the indicated time point and lysed for characterization. For immunofluorescence staining, EBs were seeded into a gelatin coated 48-well plate by day 7 and cultured for another 6 days before fixation.

\section{Teratoma formation}

Two lines of OSKME-iPSCs (OSKME-4, OSKME-5) and $\mathrm{J} 1 \mathrm{mESCs}$ were subjected to teratoma formation assay. To generate teratoma, cells were trypsinized and resuspended with serum-free culture medium. About $5 \times 10^{6}$ cells in $100 \mu$ l media were injected subcutaneously at each site near dorsal flank of anesthetized nude mice (CD-1). Three replicates were performed for each cell line. Tumors were harvested 4 weeks later and fixed in $4 \%$ formaldehyde for $24 \mathrm{~h}$ at room temperature before paraffin embedding. For histological analysis, the specimens were stained with hematoxylin and eosin (HE). The use of mice in this study was approved by Animal Care and Use Committee, Northwest A\&F University.

\section{Flow cytometry analysis}

The cells were washed once with PBS and detached with Accutase (Gibco). After centrifugation, cells were washed twice with PBS and resuspended in staining buffer $(2 \%$ FBS in PBS) for cell counting. About $1 \times 10^{6}$ cells were then transferred into $1.5 \mathrm{ml}$ Eppendorf tubes, centrifuged and incubated in $100 \mu \mathrm{l}$ staining buffer supplemented with PE anti-mouse CDH1 antibody (1 $\mu \mathrm{g}, 147303$, BioLegend) for $30 \mathrm{~min}$ on ice. The cells were washed twice and resuspended in $500 \mu \mathrm{l}$ staining buffer and analyzed with FACSCalibur (BD Biosciences). The results were analyzed with FlowJo software (Tree Star).

\section{Cell proliferation assay}

To investigate the effect of Etv 5 on cell proliferation in the reprogramming process, viable cells were counted directly. Briefly, two days after the final round of infection, cells were trypsinized and reseeded into wells of 12-well plates at a density of $5 \times 10^{3}$ cells per well, after which the cells were counted daily from day 1 to day 5 .

To investigate the effect of Etv5 on self-renewal of mESCs, cell proliferation rate was assayed using CCK-8 Cell Counting Kit (Vazyme) according to the manufacturer's instruction. Briefly, a total of $1.5 \times 10^{3}$ cells were seeded into each well of a 48-well plate, and the medium was changed every $24 \mathrm{~h}$. The CCK- 8 solution (20 $\mu \mathrm{l})$ was added to wells upon measurement, followed by $1 \mathrm{~h}$ incubation at $37^{\circ} \mathrm{C}$. The absorbance of the medium at $450 \mathrm{~nm}$ was then measured using Epoch ${ }^{\mathrm{TM}}$ Microplate Spectrophotometer (BioTek).

\section{Gene knockdown by shRNAs}

For RNAi lentivirus packaging, the lentiviral vector pSicoR-puro $(2 \mu \mathrm{g})$, psPAX2 $(1.5 \mu \mathrm{g})$ and pMD2.G $(1 \mu \mathrm{g})$ were co-transfected into HEK293T cells in $60 \mathrm{~mm}$ culture dishes using TurboFect Transfection Reagent (Thermo Scientific). The medium was changed $6 \mathrm{~h}$ later and the viral supernatant was collected at $48 \mathrm{~h}$ and filtered through $0.45 \mu \mathrm{m}$ PVDF membrane. The lentiviruses were used to infect J1 mESCs which were seeded onto gelatin coated culture dishes $24 \mathrm{~h}$ earlier. Two rounds of infection with $12 \mathrm{~h}$ interval were performed. The KSR based mESCs medium supplemented with 1.5 $\mu \mathrm{g} \mathrm{ml}^{-1}$ puromycin was used to screen the puro-resistant colonies. 


\section{RNA-seq}

Total RNA was isolated from shCtrl mESCs and Etv5KD mESCs using RNAiso Plus (TaKaRa). Two independent batches of samples were collected for RNA-seq. For strand-specific library construction and sequencing, rRNAs were removed to retain mRNAs and ncRNAs, which were then fragmented and reverse transcribed into cDNA with random primers. Second-strand cDNA were synthesized using dUTP and purified with QiaQuick PCR extraction kit (Qiagen). Then the second-strand cDNA were end repaired, poly (A) added, and ligated to Illumina sequencing adapters. UNG (Uracil-N-Glycosylase) was next used to digest the second-strand cDNA. The digested products were size-selected by agarose gel electrophoresis and amplified by PCR. The sequencing was carried on Illumina HiSeq 4000. For bioinformatics analysis, raw reads containing adapter or of low quality (Qvalue $\leq 20$ ) were removed, then mapped to the mouse reference genome (GRCm38) by HISAT2 (version 2.1.0). The resulting files were sorted using SAMtools and subjected to HTSeq (version 0.9.0) to obtain the counts of each gene. For differential expression analysis, genes abundances were quantified by $\mathrm{R}$ package edgeR. FDR $<$ 0.05 and $\log _{2}$ (fold change) $>1$ were used as the threshold to define gene expression differences as significant. GO and KEGG analysis of the differentially expressed genes were performed using the $\mathrm{R}$ package clusterProfiler ${ }^{28}$. RNA-seq data of this study can be accessed with accession number SRP111429 in NCBI's Sequence Read Archive. RNA-seq data of Tet2-KD mESCs were downloaded from GEO (GSE84458), which were processed by the same analysis procedure as Etv5-KD data did.

\section{Statistical analyses}

The statistics methods used in this study include unpaired two-tailed Student's $t$ test, one-way ANOVA with Dunnett's multiple comparisons test, and two-way ANOVA with Dunnett's multiple comparisons test, and two-way ANOVA with Sidak's multiple comparisons test. The application of each statistics method was specified in figure legends. GraphPad Prism 7.0 was used for the statistical analysis, and data are presented as means \pm SD.

\footnotetext{
Acknowledgements

We thank Dr. Anmin Lei (NWAFU, China) for kindly providing material of OG2 mice (B6; CBA-Tg(Pou5f1-EGFP) and Dr. Marius Wernig (Stanford University, USA) for generously providing Etv5 specific lentiviral shRNA vectors. We are also grateful to Wenwen Jia, Xudong Guo (Tongji University, China), and Yanting Xue (GIBH, China) for advice on miRNA research, Guangzhou Genedenovo Corp. for their help on RNA-seq. This work was supported by National Natural Science Foundation of China (31301218 to Shiqiang Zhang, 31571521 to Huayan Wang), NWAFU Fundamental Research Funds for the Central Universities (2452015038, 2452015326 to Shiqiang Zhang) and Natural Science Basic Research Plan in Shaanxi Province of China (2016JQ3004 to Shiqiang Zhang).
}

\section{Competing interests}

The authors declare no competing financial interests.

\section{Publisher's note}

Springer Nature remains neutral with regard to jurisdictional claims in published maps and institutional affiliations.

Supplementary Information accompanies this paper at https://doi.org/ 10.1038/s41419-018-0335-1.

Received: 8 November 2017 Revised: 10 January 2018 Accepted: 18 January 2018

Published online: 14 February 2018

\section{References}

1. Takahashi, K. \& Yamanaka, S. Induction of pluripotent stem cells from mouse embryonic and adult fibroblast cultures by defined factors. Cell 126, 663-676 (2006).

2. Oatley, J. M. \& Brinster, R. L. Regulation of spermatogonial stem cell selfrenewal in mammals. Annu. Rev. Cell Dev. Biol. 24, 263-286 (2008).

3. Ko, K. et al. Induction of pluripotency in adult unipotent germline stem cells. Cell Stem Cell 5, 87-96 (2009).

4. Chen, C. et al. ERM is required for transcriptional control of the spermatogonial stem cell niche. Nature 436, 1030-1034 (2005)

5. Wu, X., Goodyear, S. M., Tobias, J. W., Avarbock, M. R. \& Brinster, R. L. Spermatogonial stem cell self-renewal requires ETV5-mediated downstream activation of brachyury in mice. Biol. Reprod. 85, 1114-1123 (2011).

6. Akagi, T. et al. ETS-related transcription factors ETV4 and ETV5 are involved in proliferation and induction of differentiation-associated genes in embryonic stem (ES) cells. J. Biol. Chem. 290, 22460-22473 (2015).

7. Lujan, E. et al. Early reprogramming regulators identified by prospective isolation and mass cytometry. Nature 521, 352-356 (2015).

8. Rasmussen, K. D. \& Helin, K. Role of TET enzymes in DNA methylation, development, and cancer. Genes Dev. 30, 733-750 (2016).

9. Costa, Y. et al. NANOG-dependent function of TET1 and TET2 in establishment of pluripotency. Nature 495, 370-374 (2013).

10. Gao, Y. et al. Replacement of Oct4 by Tet1 during iPSC induction reveals an important role of DNA methylation and hydroxymethylation in reprogramming. Cell Stem Cell 12, 453-469 (2013).

11. Bermejo-Alvarez, P. et al. Tet-mediated imprinting erasure in H19 locus following reprogramming of spermatogonial stem cells to induced pluripotent stem cells. Sci. Rep. 5, 13691 (2015).

12. Buganim, Y., Faddah, D. A. \& Jaenisch, R. Mechanisms and models of somatic cell reprogramming. Nat. Rev. Genet. 14, 427-439 (2013).

13. Gregory, P. A. et al. The miR-200 family and miR-205 regulate epithelial to mesenchymal transition by targeting ZEB1 and SIP1. Nat. Cell Biol. 10, 593-601 (2008).

14. Wang, G. et al. Critical regulation of miR-200/ZEB2 pathway in Oct4/Sox2induced mesenchymal-to-epithelial transition and induced pluripotent stem cell generation. Proc. Natl Acad. Sci. USA 110, 2858-2863 (2013).

15. $\mathrm{Hu}, \mathrm{X}$. et al. Tet and TDG mediate DNA demethylation essential for mesenchymal-to-epithelial transition in somatic cell reprogramming. Cell Stem Cell 14, 512-522 (2014).

16. Sohni, A. et al. Dynamic switching of active promoter and enhancer domains regulates Tet1 and Tet2 expression during cell state transitions between pluripotency and differentiation. Mol. Cell. Biol. 35, 1026-1042 (2015).

17. de la Rica, L. et al. TET-dependent regulation of retrotransposable elements in mouse embryonic stem cells. Genome Biol. 17, 234 (2016).

18. Schrode, N., Saiz, N., Di Talia, S. \& Hadjantonakis, A. K. GATA6 levels modulate primitive endoderm cell fate choice and timing in the mouse blastocyst. Dev. Cell 29, 454-467 (2014).

19. Chazaud, C., Yamanaka, Y., Pawson, T. \& Rossant, J. Early lineage segregation between epiblast and primitive endoderm in mouse blastocysts through the Grb2-MAPK pathway. Dev. Cell 10, 615-624 (2006).

20. Takaoka, K. \& Hamada, H. Cell fate decisions and axis determination in the early mouse embryo. Development 139, 3-14 (2012). 
21. Kim, J. J. et al. Discovery of consensus gene signature and intermodular connectivity defining self-renewal of human embryonic stem cells. Stem Cells 32, 1468-1479 (2014).

22. Chen, J., Han, Q. \& Pei, D. EMT and MET as paradigms for cell fate switching. J. Mol. Cell Biol. 4, 66-69 (2012).

23. Li, X., Pei, D. Q. \& Zheng, H. Transitions between epithelial and mesenchymal states during cell fate conversions. Protein Cell 5, 580-591 (2014).

24. Samavarchi-Tehrani, P. et al. Functional genomics reveals a BMP-driven mesenchymal-to-epithelial transition in the initiation of somatic cell reprogramming. Cell Stem Cell 7, 64-77 (2010).

25. Zhou, Q., Chipperfield, H., Melton, D. A. \& Wong, W. H. A gene regulatory network in mouse embryonic stem cells. Proc. Natl Acad. Sci. USA 104, 16438-16443 (2007)
26. Kang, M., Garg, V. \& Hadjantonakis, A. K. Lineage establishment and progression within the inner cell mass of the mouse blastocyst requires FGFR1 and FGFR2. Dev. Cell 41, 496-510.e495 (2017).

27. Wamaitha, S. E. et al. Gata6 potently initiates reprograming of pluripotent and differentiated cells to extraembryonic endoderm stem cells. Genes Dev. 29, 1239-1255 (2015).

28. Yu, G., Wang, L. G., Han, Y. \& He, Q. Y. clusterProfiler: an R package for comparing biological themes among gene clusters. OMICS 16, 284-287 (2012). 